\title{
TEMA 8-2016: Sindrome de Brugada
}

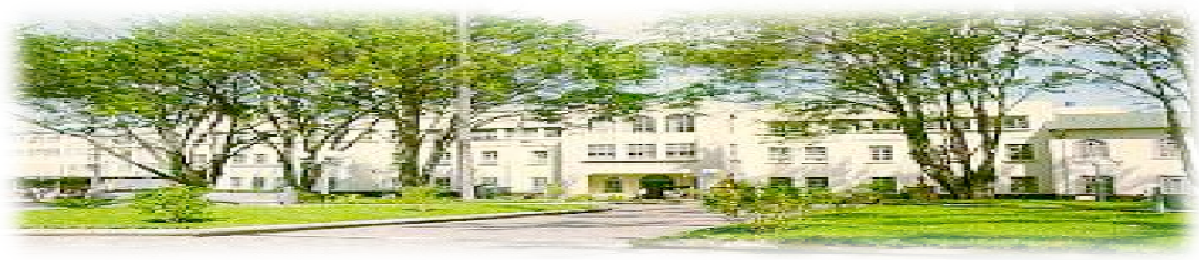

Hospital San Juan de Dios, San José, Costa Rica. Fundado en 1845

$\begin{array}{ll}\text { Recibido: } & 16 / 11 / 2015 \\ \text { Aceptado: } & 18 / 12 / 2015\end{array}$
Enrique Pacheco Ovares ${ }^{1}$
Stanley Grant Villegas

\footnotetext{
${ }^{1}$ Médico General, correo electrónico: cripacheco_17@hotmail.com

${ }^{2}$ Médico Especialista en Medicina Interna, Caja Costarricense de Seguro Social
}

\section{RESUMEN}

El Síndrome de Brugada es una de las causas más importantes de un paro cardíaco repentino y la muerte súbita cardiaca, por fibrilación ventricular. Pertenece a una serie de anomalías en el ritmo en el que se detectaron daños en la estructura cardíaca. La penetración genética variable del síndrome provoca que muchos pacientes pueden ser asintomáticos, mientras que otros pueden perecer antes del primer año de vida. La mutación de un canal de sodio es la causa directa y la mutación del gen SCN5A se relaciona directamente con este anomalías y hay otras mutaciones genéticas que se encuentran actualmente en estudio. Los medicamentos no son en realidad la primera línea de tratamiento para los pacientes sintomáticos, actualmente la ICP (desfibrilador automático implantable ) debería ser el tratamiento de primera línea para casi todos los pacientes.

PALABRAS CLAVE
Síndrome de Brugada, desfibrilador automático implantable, mutación SCN5A, canalopatía de sodio, cardiología, paro cardiaco súbito , muerte súbita cardíaca

\section{ABSTRACT}

The Brugada Síndrome is one of the most important causes of sudden cardiac arrest and sudden cardiac death, due to ventricular fibrillation. It belongs to a serie of rhythm abnormalities where no cardiac structure damage is detected. The variable genetic penetration of the syndrome causes that many patients can be asymptomatic whereas others can perish before the first year of living. The mutation of a sodium channel is the direct cause and the mutation of the SCN5A gene is directly related to this abnormalities and there are other gene mutations that are currently under study. Medications are not actually the first line of treatment for symptomatic patients, currently the ICP (implantable cardioverter- 
defibrillator) should be the first line therapy for nearly all patients.

\section{KEY WORDS}

Brugada Syndrome, implantable cardioverterdefibrillator, SCN5A mutation, sodium canalopaty, cardiology, sudden cardiac arrest, sudden cardiac death

\section{INTRODUCCIÓN}

La definición tradicional de paro cardiorrespiratorio repentino ("sudden cardiac arrest" SCA) indica el cese de la actividad cardiaca con colapso hemodinamico, típicamente debido a una taquicardia ventricular sin pulso o fibrilacion ventricular. Si el paciente fallece se le conoce como muerte súbita ("sudden cardiac death" $\mathrm{SCD})^{(1)}$

Estas condiciones ocurren frecuentemente en pacientes con enfermedad cardiovascular estructural, particularmente enfermedad coronaria.

Muchas enfermedades pueden ser consideradas como causales de SCD en pacientes donde no se encuentre una causa estructural aparente que explique, sin embargo, existen patologías hereditarias que pueden aumentar el riesgo de SCD en pacientes parientes de primer grado ${ }^{(1)}$ Dentro de las principales causas de SCD se encuentran el interevalo QT prolongado, taquicardia ventricular polimórfica con intervalo QT normal, commotio cordis, síndrome de Wolf Parkinson White y otras formas de taquicardia supraventricular, Síndrome de QT corto, fibrilación ventricular idiomática, muerte subita cardiaca familiar y de forma característica el Síndrome de Brugada; que será el que abordaremos en este artículo.

El Síndrome de Brugada es un síndrome de arritmia hereditario caracterizado por un patrón de EKG consistente en elevación del segmento ST de tipo abovedado ( $\geq 2 \mathrm{~mm}$ ), seguida de una onda $\mathrm{T}$ negativa en las derivaciones precordiales derechas de V1 a V3 (a menudo designado como patrón de EKG Brugada tipo 1), y con aumento del riesgo de muerte súbita cardiaca, por episodios de taquiarritmias ventriculares polimorfas. ${ }^{(2,}$
El grado de compromiso de este síndrome es variable, desde pacientes que cursan asintomático durante toda su vida a casos de muerte súbita cardiaca en pacientes que no llegan al primer año de vida, se considera que este síndrome afecta principalmente hombres adultos jóvenes, con una mayor incidencia en pacientes en el Sudeste asiático, con manifestaciones arritmogénicas que aparecen a una edad promedio de 40 años y en el que la muerte súbita se registra de forma habitual durante el sueño ${ }^{(4,5) \text {. }}$

Es interesante destacar que en regiones del sudeste asiático la muerte súbita nocturna es endémica, y se encuentra vinculada genética y fenotípicamente al Síndrome de Brugada. En un estudio de 30 niños efectuado en el 2007, con menos de 16 años de edad y que se encontraban afectados por el Síndrome de Brugada, fue la fiebre el principal factor precipitante más común de episodios arrítmicos, incluidos sincope y muerte súbita cardiaca ${ }^{(6)}$.

\section{DISCUSIÓN}

El Síndrome comprende una mutación autosómica dominante de penetrancia variable, cursando con hallazgos electrocardiograficos de pseudo bloqueo de rama derecha y elevación persistente del ST en las derivadas V1 a V3. Se ha realizado la definición distinguida de los pacientes que se presentan de forma asintomática y que presentan los hallazgos típicos en el electrocardiograma, condición que se clasifica como Patrón de Brugada. En los pacientes donde ya hayan experimentado muerte súbita cardiaca o taquiarritmia ventricular sostenida, o que asocian al menos uno o más criterios clínicos, se clasifica como Síndrome de Brugada ${ }^{(7) .}$ A pesar de esta distinción, en el trazo electrocardiográfico no hay diferencia en cuanto a los hallazgos.

Como se indicó anteriormente existen tres patrones electrocardiográficos característicos del Síndrome de Brugada pero se ha llegado a un consenso en donde a partir del trazo del ST se puede clasificar el Síndrome de Brugada en dos tipos: el síndrome de brugada clásico o tipo 1 $\mathrm{EKG}$, donde se encuentra la elevación del $\mathrm{ST} \geq$ $2 \mathrm{~mm}$, el cual desciende con una convexidad hacia arriba hacia una onda $\mathrm{T}$ invertida $\mathrm{y}$ un 
patrón tipo 2 (que es en realidad una combinación de los tipos 2 y 3 descritos anteriormente por otros autores), en donde el segmento ST presenta una "montura" en la configuración de la onda ST-T, en la cual el segmento ST elevado desciende hacia la línea isoeléctrica, para luego ascender de nuevo hacia una onda $\mathrm{T}$ vertical $\mathrm{o}$

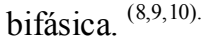

Se estima que entre el 20 y $30 \%$ de los casos de Síndrome de Brugada corresponden a mutaciones de pérdida de función en el canal cardiaco del sodio, codificado por el gen SCN5A, y son clasificados como Síndrome de Brugada tipo 1 (11). En 2010, un compendio internacional de mutaciones en SCN5A, en pacientes derivados para ser sometidos a pruenas geneticas por el Síndrome de Brugada, notifico casi 300 mutaciones diferentes en 438 de 2.111 (21\%) pacientes no relacionados, y la deteccion de mutaciones osciló entre el 11 y el $28 \%$ en nueve centros ${ }^{(12)}$.

\section{Diagnóstico Diferencial:}

Dentro de los principales diagnósticos diferenciales del Síndrome de Brugada se encuentran otras condiciones que cursan con alteraciones en la conducción y anormalidades del segmento ST en las derivadas V1 a V3, como lo seria desde luego isquemia aguda miocárdica o infarto agudo de miocardio, hipotermia, pericarditis aguda, repolarización temprana, cardiomiopatía arritmogénica ventricular derecha y bloqueo atípico de la rama derecha ${ }^{(7)}$.

\section{Pronóstico:}

Sin duda los factores pronósticos mas importantes vienen siendo la presencia de taquiarritmias ventriculares que lleven a parto cardiorrespiratorio súbito o bien sincope, otros factores menos fuertes para predicción de eventos futuros son la fibrilación atrial, sexo masculino y antecendentes heredofamiliares de SCA. Ondas J anómalas en las derivaciones inferolaterales, seguidas de un segmento ST de morfología plana u horizontalizada (opuesta a la morfología ascendente) se asocia con una mayor incidencia de eventos cardiacos en una población de 460 pacientes sintomáticos y asintomáticos con Síndrome de Brugada ${ }^{(13) .}$

\section{Tratamiento:}

El tratamiento para el Síndrome de Brugada se enfoca básicamente en la prevención de cualquier arritmia ventricular con la implantación de cardiovertor-desfibrilador implantable (ICD). Inicialmente el tratamiento farmacológico se ha probado con un éxito reducido, de esta manera el uso de ICD se considera el tratamiento de primera línea para casi todos los pacientes. Es importante mencionar que pacientes que curse con el patrón de Brugada por electrocardiograma sin ningún otro síntoma, no requieren ninguna terapia especifica ${ }^{(7)}$.

Se ha demostrado cierto beneficio con el uso de quinidina, la potencial eficacia de este medicamento se ilustró en un reporte, donde se le administró el medicamento a 25 pacientes con Síndrome de Brugada de los cuales 15 estaban sintomáticos y 10 asintomáticos; posteriormente al tratamiento de bisulfato de quinidina con una dosis media de $240 \mathrm{mg} /$ día. El tratamiento se continuó en 19 pacientes por una media de 56 meses, ninguno de estos pacientes presentaron eventos arrítmicos, a pesar de esto, dos tuvieron un sincope no relacionado a arritmia ${ }^{(14)}$.

Otros medicamentos antiarrítmicos de I Clase pueden crear efectos, especialmente los bloqueadores de canales de sodio como lo son flecainida, ajmalina o procainamida pueden transitoriamente inducir cambios característicos del EKG de tipo 1. Amiodarona es el agente más efectivo para la prevención de taquiarritmias ventriculares a pesar que tiene muchos efectos adversos potenciales con su uso, que con otros medicamentos antiarrítmicos ${ }^{(7)}$. El empleo de cilostazol, un inhibidor de la fosfodiesterasa que evita la agregación plaquetaria y es aprobado para el tratamiento de la claudicación intermitente de la marcha, puede presentar un aporte beneficioso en pacientes con Síndrome de Brugada al mediar el aumento de calcio intracelular y aumentando la frecuencia cardiaca. ${ }^{(15)}$

\section{CONCLUSIONES}

- El Síndrome de Brugada corresponde una de las principales causas de SCA y $\mathrm{SCD}$ en pacientes jóvenes 
- Actualmente el tratamiento farmacológico ha sido desplazado por el empleo de ICD

- La penetrancia genética de este trastorno, crea un rango muy amplio dentro del espectro clínico, donde existen pacientes que pueden cursar asintomáticos durante toda su vida y aquellos que pueden fallecer antes del año de vida.

- Hallazgos específicos en el electrocardiograma nos permiten crear una sospecha clínica de que el paciente puede presentar el síndrome, es importante realizar diagnósticos diferenciales respectivos.

- Característicamente afecta a pacientes masculinos y jóvenes, en el sudeste asiático se ha convertido en una patología endémica.

\section{BIBLIOGRAFÍA}

1. Mark S Link: Sudden cardiac arrest in the absence of apparent structural heart disease. Uptodate, 2015.

2. Chen PS Priori SG. The Brugada Syndrome, Journal American College of Cardiology 51:1176, 2008.

3. Brugada P Benito B Brugada R Brugada J: Brugada Syndrome: Update 2009, Hellenic Journal of Cardiology 50:352, 2009.

4. Shimizu W: Clinical impact of genetic studies in lethal inherited cardiac arrhytmias, Cir J 72:1926, 2008.

5. Ruan Y Liu N Priori SG: Sodium channel mutations and arrhytmias; Nat Rev Cardiol 6:337, 2009.

6. Probst V Denjoy I Meregalli PG et al: Clinical aspects and prognosis of Brugada Syndrome in children, Circulation 115: 2042, 2007

7. V. Wylie J C. Garlitski A: Brugada Syndrome. Up to date, 2015

8. Wilde AA Antzelevitch C Borggrefe M et al: Proposed diagnostic criteria for the Brugada Syndrome. Europe Heart Journal 2002; 23:1648

9. Antzelevitch C Brugada P Borggrefe M et al: Brugada Syndrome: report of the second consensus conference: endorsed by the Heart Rhythm Society and the
European Heart Rhythm Association. Circulation 2005; 111:659

10. Bayés de Luna A Brugada J Baranchuk A et al: Current electrocardiographic criteria for diagnosis of Brugada pattern: a consensus report. J. electrocardioly 2012; 45:433

11. Braunwald

12. Kapplinger $\mathbf{J}$ Tester D Aldeas $\mathrm{M}$ et al: An internacional compendium of mutations in the SNC5A-encoded cardiac sodium channel in patients referred for Brugada sindrome genetic testint, Heart Rhythm 7:33, 2010.

13. Takagi M Aonuma K Sekiguchi $\mathrm{Y}$ et al: The prognostic value of early repolarization ( $J$ wave) and ST segment morphology after $J$ wave in Brugada Syndrome: multicenter study in Japan. Heart Rhythm 2013; 10:533.

14. Yan GX Antzelevitch C. Cellular basis for the Brugada syndrome and other mecanisms of arrhythmogenesis associated with ST segment elevation. Circulation 1999; 100:1660

15. Tsuchiya Y Ashikaga K Honda T Arita M. Prevention of ventricular fibrillation by cilostazol, an oral phosphodiesterase inhibidor, in a patient with Brugada syndrome. J Cardiovascular Electropysiology 2002; 12:698

\section{CONFLICTOS DE INTERES}

Los autores declaran que no existe ningún conflicto de interés. 